\title{
MODELO TEÓRICO DE LA CALIDAD DEL SERVICIO INTERNO EN LAS EMPRESAS TURÍSTICAS DESDE LA PERSPECTIVASOCIALSUSTENTABLE
}

\author{
Aralí Larios Calderón* \\ Alfonso González Damián**
}

Resumen: Estudios recientes acerca de la calidad del servicio interno en la empresa han sugerido la necesidad de dirigir la investigación hacia la identificación de variables que ayuden a comprender qué está afectando el comportamiento de los empleados dentro de la organización. Por lo anterior, se planteó como objetivo del presente documento, presentar un modelo teórico para el análisis de la calidad del servicio interno desde una perspectiva social sustentable, en el campo del turismo con énfasis en tres categorías de interés: condiciones laborales, situaciones personales y contexto local del trabajador. La metodología se basó en tres etapas: en la primera, se realizó una revisión literaria en journals de alto impacto sobre el concepto de calidad del servicio interno; la segunda se enfocó en la consulta de libros base de las tres disciplinas identificadas en la primera etapa que datan desde 1998 hasta el 2014. Por último, mediante un ejercicio analítico - sintético se organizaron de manera sistemática los conceptos y fundamentos definidos, determinando el diseño del modelo. Se concluye que la propuesta del modelo teórico es una visión que pretende integrar variables de diversas disciplinas, matizadas como categorías analíticas, en el marco del desarrollo social sustentable para las empresas turísticas.

Palabras clave: Calidad del servicio interno; Modelo teórico; Empresas turísticas.

\section{MODELOTEÓRICO DE LA CALIDAD DEL SERVICIO INTERNO EN LASEMPRESASTURÍSTICAS DESDE LA PERSPECTIVASOCIAL SUSTENTABLE}

Resumo: Estudos recentes sobre a qualidade do serviço interno na empresa têm sugerido a necessidade de direcionar a pesquisa para identificar as variáveis que ajudam a compreender o que afetar o comportamento dos funcionários dentro da organização. Por isso, este artigo visa apresentar um modelo teórico para analisar a qualidade do serviço interno de uma perspectiva social sustentável na área do turismo com ênfase em três categorias de interesse: as condições de trabalho, situações pessoais e contexto local do trabalhador. A metodologia foi baseada em três etapas: 1) uma revisão da literatura foi realizada em periódicos de alto impacto sobre o conceito de qualidade do serviço interno; 2) consulta de banco de dados para todas as três disciplinas identificados na primeira fase de namoro de 1998 a 2014; e 3) através do exercício analítico - sintéticos conceitos e princípios sistematicamente organizados definido, determinando o design modelo. Conclui-se que o modelo teórico proposto é uma visão que tem como objetivo integrar variáveis de várias disciplinas, nuances como categorias de análise, no âmbito do desenvolvimento social sustentável para as empresas de turismo.

Palavras-chave: qualidade interna serviço, modelo teórico, empresas de turismo.

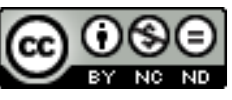

Licenciada por Creative Commons Atribuição Não Comercial / Sem Derivações/4.0/Internacional

\section{THEORETICALMODEL OF THE INTERNALSERVICE QUALTY IN TOURISM COMPANIES FROM THESUSTAINABLE SOCIAL PERSPECTIVE}

\begin{abstract}
Recent studies on the quality of internal service in the company have suggested directing research toward identifying variables that help understand what is affecting employee behavior within the organization. Therefore, the objective of the research was to design a theoretical model that allows analysis of the quality of internal service from the perspective of social sustainability. It was analyze the field of tourism in three categories: labor conditions, personal situations and local context of the worker. The methodology was based on three stages: in the first, a literary review was carried out in journals of high impact on the concept of quality of the internal service; The second focused on the consultation of basic books of the three disciplines identified in the first stage that date from 1998 to 2014 . Finally, through an analyticsynthetic exercise, the concepts and foundations were defined in a systematic way, determining the design of the model. It is concluded that the proposal of the theoretical model is an integrative vision with variables of each discipline delimited in the categories of analysis, within the framework of sustainable social development for tourism companies.
\end{abstract}

Keywords: Internal service quality, theoretical model, tourism companies.

* Maestría en Turismo y Dirección Hotelera. Profesor investigador en la Universidad Tecnológica de la Riviera Maya/México. Integrante del Cuerpo Académico de Estudios Empresariales en Turismo, con líneas de investigación en la calidad del servicio y competencias profesionales. Universidad Tecnológica de la Riviera Maya. Av Paseo del Mayab 4000 Región 79 Playa del Carmen, Solidaridad, Quintana Roo, C.P. 77710. Phone: 984877 4600. [arali.larios@utrivieramaya.edu.mx]

** Doctor en Ciencias Sociales y Políticas (2007) por la Universidad Iberoamericana, Ciudad de México. Maestro en Administración (1997) y Licenciado en Turismo (1991), ambos por la Universidad Autónoma del Estado de México. Profesor investigador en la Universidad de Quintana Roo Unidad Académica Cozumel, integrante del Cuerpo Académico de Estudios Ambientales, con líneas de investigación centradas en temas de sociología constructivista del turismo y la gestión socialmente sustentable del turismo. Investigador nacional (SNI nivel I). Universidad de Quintana Roo, Campus Cozumel/México. AvAndrés Quintana Roo s/n Col. San Gervasio, Cozumel, 77600. Phone: 987 8729000. [gonzalezd@uqroo.edu.mx] 


\section{INTRODUCCIÓN}

La investigación acerca de la calidad del servicio interno ha conseguido demostrar su importancia para las empresas, puesto que repercute en la satisfacción del cliente y con ello en la posibilidad de que se mantenga fiel a la firma, lo que a su vez impulsa la rentabilidad sostenida. Reviste interés particular para las empresas turísticas, al ser proveedoras de servicios y al medir gran parte de su desempeño a través de la satisfacción del cliente.

El interés académico por analizar los antecedentes, factores, consecuentes y posibilidades de la calidad en el servicio interno, así como sus conexiones con el desempeño de la empresa, la satisfacción del recurso humano y la satisfacción del cliente se ha conducido desde distintas perspectivas, destacandose los trabajos desde las disciplinas económico administrativas, particularmente el marketing pero con relevantes aportaciones desde perspectivas de las ciencias de la conducta humana, tanto desde la psicología como desde la sociología.

Estudios recientes han sugerido la necesidad de dirigir la investigación hacia la identificación de variables que ayuden a comprender qué está afectando el comportamiento de los empleados dentro de la organización (Pantouvakis y Mpogiatzidis, 2013; Chen, 2013; Sharma, Chuen y Kingshott, 2016).

Por lo anterior, se planteó como objetivo del presente documento, presentar un modelo teórico para el análisis de la calidad del servicio interno desde una perspectiva social sustentable, en el campo del turismo con énfasis en tres categorías de interés: condiciones laborales, situaciones personales y contexto local del trabajador.

El carácter de la investigación es descriptivo, utilizando el método analítico y sintético para el desarrollo del trabajo. La metodología se basó en tres etapas: en la primera, se realizó el estado del arte del concepto calidad del servicio interno a través de la revisión de journals de alto impacto, organizando los artículos en dos compendios: el primero que hacía referencia a las investigaciones de las dimensiones de la calidad del servicio interno, y el segundo, sobre estudios enfocados a comprobar la importancia de la calidad del servicio interno en la empresa, incorporando en su estudio variables conceptuales relacionadas, encontrando así disciplinas que estudiaban dicho constructo desde su enfoque.
La segunda etapa se enfocó en la consulta de libros base de las tres disciplinas identificadas en la primera etapa (económica - administrativa, marketing y sociología de las empresas) que datan desde 1998 hasta el 2014, a través de una consulta directa y libros accesibles en google academic.

Por último, mediante un ejercicio analítico sintético se organizaron de manera sistemática los conceptos y fundamentos definidos, aunados a las necesidades actuales en el estado del arte del concepto, determinando así, el diseño del modelo.

Se concluye que la propuesta del modelo teórico es una visión que pretende integrar variables de diversas disciplinas, matizadas como categorías analíticas, en el marco del desarrollo social sustentable para las empresas turísticas.

\section{MARCO TEÓRICO}

\subsection{Calidad del Senvicio Interno: orígenes y dimensiones}

El concepto de calidad del servicio interno ha cobrado interés en la investigación, la búsqueda por apoyar al crecimiento y desarrollo de las empresas que ofertan servicios, y atender su naturaleza de ser productivas y rentables, ha llevado al reconocimiento de la importancia del recurso humano y su satisfacción.

A la fecha el turismo es un sector clave para el progreso socioeconómico de los países por su creación de puestos de trabajo y de empresas así como también por el desarrollo de infraestructuras con un impacto en el destino que se promociona (Peinado y García, 2016).

En 1994, se identifica la aparición del término calidad del servicio interno ó internal service quality en la cadena de servicio - beneficio de Heskett y otros (1994), modelo causal enfocado al crecimiento de los ingresos y ganancias de la empresa a través de la integración de variables para el desarrollo organizacional en el contexto de la industria del servicio. Dicho modelo se basó sobre los supuestos de que la calidad del servicio interno conduce a empleados satisfechos, los cuales permiten la entrega de un servicio con alto valor, resultando en clientes satisfechos, provocando con ello su lealtad, y conduciendo a la producción de ganancias y crecimiento para la empresa. 
El resultado de dicho modelo en el campo de la investigación generó una serie de estudios para analizar las diferentes problemáticas asociadas con los argumentos expuestos, ya que el mismo autor planteó la necesidad de examinar las relaciones de causalidad entre las variables, lo que a su vez requeriría de una mayor fundamentación (Yee, Yeung y Cheng, 2011).

Es de enfatizar que el principio básico del concepto de calidad del servicio interno es el concepto de servicio al cliente interno, el cual consiste en que "cada departamento (o a veces trabajadores) recibe trabajo de y/o desarrolla trabajo para otro departamento (otro trabajador). El departamento o empleado que desarrolla el trabajo es el productor del servicio interno y el departamento o empleado que recibe es el cliente interno; su interacción es el encuentro del servicio interno (Gunawardane, 2009).
Por lo que la presencia del concepto de calidad del servicio interno está en el encuentro que tienen los departamentos o el recurso humano de la organización en el desempeño del servicio que entre ambos se proveen" (Larios y González, 2017).

En el 2009, Gunawardane identificó los estudios dirigidos a indagar sobre las dimensiones que componen la calidad del servicio interno que datan de 1994 al 2003, encontró que la mayoría de estos estudios han usado la clasificación de las dimensiones de la calidad del servicio externo de Parasuraman y otros (1998) para ello.

A la fecha se siguen investigando las dimensiones de la calidad del servicio interno (ver tabla 1), ya que los estudios han revelado que es un concepto complejo por la cantidad de variables que intervienen en su comprensión.

Tabla 1: Estudios sobre las dimensiones de la calidad del servicio intemo.

\begin{tabular}{|c|c|c|c|}
\hline Autor & Campo de estudio & Objetivo & $\begin{array}{l}\text { Elementos, Dimensiones o componentes } \\
\text { encontrados }\end{array}$ \\
\hline Wildes (2007) & $\begin{array}{l}\text { Industria de } \\
\text { alimentos y bebidas }\end{array}$ & $\begin{array}{l}\text { Examinar la capacidad de la calidad del } \\
\text { servico interno para moderar el } \\
\text { puesto estereotipado de los } \\
\text { empleados de los restaurantes. }\end{array}$ & $\begin{array}{l}\text { Behavioral intent (intent to stay and intent } \\
\text { to recommend) }\end{array}$ \\
\hline Jun \& Cai (2010) & $\begin{array}{l}\text { Departamento de } \\
\text { compras }\end{array}$ & $\begin{array}{l}\text { identificar si los clientes internos } \\
\text { perciben la calidad del servicio } \\
\text { significativamente y positivamente } \\
\text { relacionada con su satisfacción }\end{array}$ & 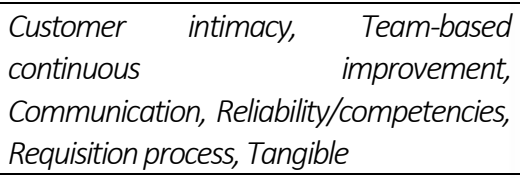 \\
\hline $\begin{array}{l}\text { Vanniarajan y } \\
\text { Subbash (2011) }\end{array}$ & $\begin{array}{l}\text { Servicio financiero } \\
\text { (bancos) }\end{array}$ & $\begin{array}{l}\text { Identificar los aspectos importantes de } \\
\text { la calidad del servicio interno. }\end{array}$ & $\begin{array}{l}\text { Employees' and customers' orientation, } \\
\text { team orientation and employee } \\
\text { orientation, learning environment and } \\
\text { outcome orientation }\end{array}$ \\
\hline $\begin{array}{l}\text { Pantouvakis } \\
\text { (2011) }\end{array}$ & $\begin{array}{l}\text { Servicios } \\
\text { hospitalarios }\end{array}$ & $\begin{array}{l}\text { Investigar la percepción de los líderes } \\
\text { de los servicios hospitalarios en el } \\
\text { contexto del sistema de salud público } \\
\text { de Grecia. }\end{array}$ & $\begin{array}{l}\text { Job satisfaction, internal service quality } \\
\text { physical characteristics, internal service } \\
\text { quality interactive characteristics } \\
\text { (empowermentand continuous learning) }\end{array}$ \\
\hline Chen (2013) & $\begin{array}{l}\text { Industria hotelera } \\
\text { internacional }\end{array}$ & $\begin{array}{l}\text { Construir un modelo de las influencias } \\
\text { sobre la calidad del servicio interno. }\end{array}$ & $\begin{array}{l}\text { Bureaucratic organizational culture and } \\
\text { leadership }\end{array}$ \\
\hline $\begin{array}{l}\text { Umamaheswari } \\
\text { (2014) }\end{array}$ & $\begin{array}{l}\text { Industria } \\
\text { manufacturera }\end{array}$ & $\begin{array}{l}\text { 1.- Examinar las dimensiones de la } \\
\text { calidad del servicio interno. } \\
\text { 2.- Identificar los factores afectando la } \\
\text { calidad del servicio interno. }\end{array}$ & $\begin{array}{l}\text { 1.- Individual, Departmental \& } \\
\text { organizational dimensions. } \\
\text { 2.- Roles and responsibility, service } \\
\text { competency, communication, resolution } \\
\text { and alignmentfactors. }\end{array}$ \\
\hline $\begin{array}{l}\text { Sharma, Chuen y } \\
\text { Kingshott(2016) }\end{array}$ & $\begin{array}{l}\text { Industria } \\
\text { manufacturera }\end{array}$ & $\begin{array}{l}\text { La calidad del servicio interno tiene un } \\
\text { efecto positivo sobre la satisfacción del } \\
\text { empleado, compromiso y bienestar } \\
\text { del trabajador. }\end{array}$ & $\begin{array}{l}\text { Employee satisfaction } \\
\text { Employee commitment } \\
\text { Employee well-being }\end{array}$ \\
\hline $\begin{array}{l}\text { Fadil, Singh y } \\
\text { Joseph (2016) }\end{array}$ & $\begin{array}{l}\text { Oficina de servicios } \\
\text { públicos (gobierno) }\end{array}$ & $\begin{array}{l}\text { Comprender mejor la influencia de la } \\
\text { innovación organizacional en la } \\
\text { calidad del servicio interno. }\end{array}$ & $\begin{array}{l}\text { Innovation organizational (Innovation } \\
\text { leadership, managerial levers and business } \\
\text { process) }\end{array}$ \\
\hline
\end{tabular}

Nota: Para mantener el concepto original de las palabras, no se hizo la traducción de las dimensiones, elementos o componentes.

Fuente: Elaboración propia con información de Larios y González (2017). 
Con el paso del tiempo la definición de calidad del servicio interno se ha ido complementando con los diferentes puntos de vista de los investigadores. De ser conceptualizada solo como un ambiente y ser percibida a través de los sentimientos del trabajador hacia su trabajo, sus colegas y la empresa (Heskett,1994), a ser considerada en una perspectiva administrativa: como el resultado de la implementación de la alta calidad de los servicios de apoyo y las políticas de la organización, que posibilitan que los empleados produzcan resultados en términos de calidad del servicio y valor al cliente (Lings, 2004), y desde el punto de vista del marketing interno: abarcando todos los factores que contribuyen a la satisfacción del empleado y la filosofía de la cadena de valor de Heskett (1994), enlazando el aumento y la creación de valor al cliente (Pantouvakis y Mpogiatzidis, 2013).

Los diferentes ángulos de las investigaciones sobre la calidad del servicio interno, han derivado en estudiar al concepto desde un enfoque multidisciplinar (Wildes, 2007; Sharma, Chuen y Kingshott, 2016). Las revisiones teóricas parten de tres conceptos: satisfacción del cliente, cliente interno y calidad del servicio, considerando como parte del análisis, al contexto en el cual se desarrolla la investigación.

A la par de las investigaciones que estudian las dimensiones que componen el concepto, se identifica una línea de investigación sobre cómo evaluar o medir la calidad del servicio interno, con una serie de estudios centrados en el diseño de modelos o instrumentos con tal finalidad: INTQUAL (Caruna y Pitt, 1997), INTERSERVQUAL (Frost y Kumar, 2000), MECSI (Balmori y Flores, 2014).

Las tendencias en las investigaciones más recientes, han sugerido la necesidad de indagar con enfoque cualitativo respecto a la percepción de los empleados, incluyendo variables sociodemográficas y aquellos factores que ayuden a comprender qué está afectando el comportamiento de los empleados dentro de la organización, incluso variables exógenas a la empresa, adhiriendo al concepto de satisfacción del empleado, el de bienestar y calidad de vida.

Hoy en día es importante que el turismo no solo crezca en cuestiones económicas para los paísesmayor infraestructura y mayor promoción-, sino que el crecimiento vaya de la mano del desarrollo humano del recurso que utiliza para brindar el servicio, por lo que los estudios cualitativos aportan las experiencias de vida de las personas y su bienestar dentro de la actividad turística (Croes y Rivera, 2015).

\subsection{Evolución de los Estudios sobre Calidad del Servicio Segundo Diferentes Perspectivas}

\subsubsection{La calidad del senvicio interno desde diversas perspectivas disciplinares: económico-administrativa, marketing y sociología de las organizaciones}

Las investigaciones publicadas han permitido avanzar en la comprensión del concepto calidad del servicio interno, identificando como resultado que su tratamiento ha sido como objeto e instrumento de estudio, dejando como referencia la importancia de indagar a profundidad en aspectos del comportamiento humano. Por lo que para abordar en el campo de la investigación científica el análisis del concepto y contribuir al conocimiento, se determinaron perspectivas que reflejan las investigaciones analizadas en el estudio del arte, y que permitieran ahondar en la comprensión del constructo, en una primera instancia, desde una perspectiva social atendiendo las necesidades actuales en la investigación, concluyendo de esta manera en una clasificación de tres enfoques: perspectiva económica - administrativa, perspectiva del marketing y perspectiva de la sociología de las organizaciones. A continuación, el análisis de la relación de las perspectivas disciplinares y el concepto de calidad del servicio interno:

\section{A) Perspectiva económico - administrativa}

Cómo se mencionó, la calidad del servicio interno como objeto de estudio, ha integrado variables conceptuales de diferentes disciplinas que han ayudado a comprender el concepto. Estos estudios han dejado ver que la filosofía del concepto, es considerar al recurso humano dentro de la organización como parte importante en la productividad y rentabilidad de la empresa, por lo que la satisfacción en el trabajo es sustancial (Pantouvakis, 2011).

Soportando lo anterior, se encuentran los estudios que surgen a partir de los supuestos de la cadena de Heskett (1994) enlazando el clima organizacional y la satisfacción de los empleados con los afectos en la satisfacción del cliente y por ende las ganancias de la empresa (Anderson, Fornell y 
Lehmann, 1994;Yee, Yeung y Cheng, 2008; Chi y Gursoy, 2009; Kralj y Solnet, 2010).

Aunado a lo anterior, aparece la integración de la calidad en los procesos productivos de la empresa, en un inicio dirigida hacia el control dentro del proceso administrativo con el objetivo de detectar errores o defectos, y después, conduciéndola como un sistema administrativo de mejora continua enfocada hacia el cliente (Chiavenato, 2014), implicando en el campo de la investigación, agregar la calidad como sistema dentro de la administración en una organización, corroborando la correlación existente entre la satisfacción de los clientes internos y externos, y el aumento de la productividad de la organización, por lo que con ello se agrega a la gestión de la calidad, elementos para la formación del recurso humano (Finn, Baker, Marshall, Anderson, 1996; Romero y Ledo, 2008).

Teniendo como hilo conductor la satisfacción del trabajador, en la revisión literaria sobre administración, se detectó que las teorías con mayor relación en el tema, son las que abarcan las necesidades de las personas, y que la motivación y el liderazgo, son fuente de teoría para su comprensión. Pero además se identificó que la forma de administración que más soporta trabajar dentro del esquema del concepto de calidad del servicio interno, son: la administración humanística, por su enfoque hacia el recurso humano y la aplicación de la psicología del trabajo en la administración para comprender el comportamiento en lo individual y lo colectivo del recurso humano (Chiavenato, 2014); el enfoque sistémico "al considerar a las organizaciones como entidades complejas que responden a un comportamiento y disponen de mecanismos de control y adaptación al medio" (De Val, 1997, p. 83), por lo que considera los factores internos y externos del pensamiento administrativo como un todo integrado (Johnson, 1963 citado en Rue y Byars, 2013), motivando al administrador a ver los aspectos humanos como un todo que produce sinergia, buscando producir teorías y formulaciones conceptuales que puedan crear condiciones de aplicación práctica (Luna, 2015); y por último, el enfoque de administración por calidad total, en donde la organización entendida como un sistema, parte del enfoque al cliente, estableciendo al interior una cultura de participación, reciprocidad, trabajo en equipo, planeación y mejoramiento continuo.
En este modelo de administración, se busca satisfacer tanto clientes internos y externos basados en principios de calidad total, en los que mediante el liderazgo se determina el rubro y la cultura deseada al establecer los planes y proyectos estratégicos necesarios para colocar a la organización en un nivel de competencias que garantice su permanencia y crecimiento (Cantú, 2011).

Por lo que en la búsqueda de la comprensión de la calidad del servicio interno, se puede recurrir a los enfoques: humanístico, sistemático y de calidad en la administración, sin dejar a un lado que se tiene que seguir manteniendo la naturaleza de las empresas de ser productivas y rentables, pero de la mano de la satisfacción de los empleados a través de atender sus necesidades provocadas por el mismo entorno interno o externo de la organización, utilizando principalmente teorías de motivación y liderazgo para ello.

\section{B) Perspectiva del marketing}

La perspectiva más cercana para analizar el concepto de calidad del servicio interno, no solo como objeto de estudio sino también como instrumento, es el marketing, a través de la calidad del servicio y el marketing interno. En lo que respecta a la calidad del servicio, en un primer plano, su filosofía funge como soporte para el estudio de la calidad del servicio interno, "estableciendo que mejorar la calidad del servicio interno, contribuye a mejorar la calidad del servicio al cliente, y por ende el desempeño financiero de la empresa (Anderson et al., 1994; Capon, Farley y Hoening, 1990; Zeithaml, 2000; Rust et al., 2000)" (Larios y González, 2017). Además se resalta que con la aplicación de este concepto, las organizaciones trascienden de tener una ventaja competitiva, a alcanzar una ventaja competitiva sustentable (Pfau et al., 1991; Albrecht, 1993 citados en Vanniarajan y Subbash, 2011).

En un segundo plano, los estudios (Large y Köning, 2009) evalúan la calidad del servicio interno principalmente con el modelo SERVQUAL de Parasuraman, Zeithmal y Berry (1985), utilizando asimismo dicha metodología para diseñar instrumentos más acordes a una evaluación interna de la calidad del servicio, generándose el INTQUAL (Internal Measure of Service Quality) de Caruna y Pitt (1997), el INTERSERVQUAL (Internal Service Quality) de Frost y Kumar (2000) y el MECSI (Evaluation model for internal service quality) de Balmori y Flores (2014). 
En relación al marketing interno, su relación reviste en el cliente interno, ya que el marketing interno considera al recurso humano como cliente interno, siendo éste toda persona que trabaja en una organización y es al mismo tiempo, un proveedory un cliente, por lo que la preocupación de esta disciplina es lograr que el personal comprenda, tanto a nivel individual como entre departamentos, que hay clientes dentro de la propia organización, y que en consecuencia, debe preocuparse por determinar lo que puede hacer para elevar el nivel y la calidad de los servicios que ofrece a esos clientes (Christopher, Payne y Ballantyne, 1994).

Conjuntamente se soporta la idea, de que las empresas deben de ofrecer trabajos de acuerdo a las necesidades y requerimientos de los empleados (Sasser y Arbeit 1976; To, W., Martin, E., y Yu T., 2015; Gounaris, S., 2006; Turkoz y Akyol, 2008).

Por lo que de la misma forma que en la administración, en el campo de la investigación (Scheider, B., Bowen, E., 1993 citados en Dumitrescu, Cetina y Pentescu, 2012), el marketing interno abre una brecha sobre la premisa de que la satisfacción del cliente interno afecta la satisfacción del cliente, y por ende la productividad de la empresa.

Entonces, aunado a los elementos de la perspectiva económica administrativa, se puede agregar a la comprensión del constructo de la calidad del servicio interno: la filosofía de la calidad del servicio y las metodologías para su medición, así como la filosofía del marketing interno y sus herramientas para alcanzar empleados satisfechos

\section{C) Perspectiva de la sociología de las organizaciones}

La cantidad de variables involucradas en el estudio de la calidad del servicio interno, así como su tendencia por investigar a profundidad los factores que afectan el comportamiento del recurso humano, hacen ver al concepto holístico y complejo, principalmente porque son personas las involucradas en la interacción de la calidad del servicio.

Por lo anterior se piensa en la sociología de las organizaciones como pilar para el estudio del concepto, ya que esta disciplina considera a la organización como un ente social, es decir, la vida en grupo de los seres humanos y su comportamiento social resultante (Velázquez, 2008).
La sociología de las organizaciones considera que las agrupaciones sociales en una organización implican la coordinación de tareas y por ende un ambiente de cooperación entre ellos guiados por la dirección o gerencia para alcanzar los resultados establecidos por la empresa, por lo que este tipo de organización social afecta a los miembros de la empresa moldeando su actitud y comportamiento. Al mismo tiempo, la mejora en la productividad de la empresa, así como la calidad de los productos y servicios que ofrecen, requieren de una alta participación de los empleados, por lo que su actitud y comportamiento involucra la reflexión de lo que hacen y su compromiso con su trabajo (Robbins, 1998).

Scott's (1998 citado en Handel, 2003), divide las investigaciones sobre la sociología de las organizaciones en: racional, natural y sistemas abiertos, por su conceptualización, se identifican la tipología natural y de sistemas abiertos para estudiar la calidad del servicio interno, dado que la primera no solo considera a la organización como una estructura racionalmente construida para lograr objetivos específicos, sino que también la ve como un sistema social y humano, y la segunda, pone atención en la estructura interna y su funcionamiento con las organizaciones sociales de ambientes externos analizando su efecto al interior.

Al ser entonces las organizaciones entes sociales y abiertos, se analiza a la empresa desde las personas, las cuáles constituyen el sistema social interno de la organización. Las personas son los seres que viven, piensan, sienten, y trabajan en la organización para alcanzar sus objetivos. En particular, la fuerza laboral adquirió una gran diversidad, lo que significa que los empleados aportan una amplia gama de antecedentes educativos, talentos y perspectivas a su empleo (Newstrom, 2011).

Las personas en una organización son un importante bloque de construcción de la sociedad, son un generador de la estructura social, es difícil no pensar que las organizaciones afectan su vida, ya que pasan mucho tiempo dentro de ellas, y no solo afectan a las personas como tal, sino también la distribución del dinero, del poder y de la felicidad de la sociedad fuera de la organización (Handel, 2003). Además, el ambiente externo de una organización influye en las actitudes y condiciones de trabajo de las personas, por lo que el ambiente externo debe de 
incluirse en el estudio de la conducta humana en las organizaciones (Newstrom, 2011).

Al mismo tiempo, es de observarse que las conductas de las personas dentro de la empresa, son diferentes a las conductas individuales y grupales. Las acciones más representativas en una organización son las de un colectivo ya que "los individuos rara vez trabajan solos, aislados de los demás miembros de la organización, generalmente los trabajadores trabajan en grupos" (Gibson, Ivancevich, Donnelly, Konopaske, 2009, p. 10). Derivado de esas acciones, se generan grupos formales e informales: los primeros, son "los grupos creados por las decisiones administrativas para alcanzar las metas establecidas por la organización" (Gibson et al., 2009, p. 228), los segundos, "son agrupaciones naturales de personas en una situación laboral que se reúnen en respuesta a necesidades sociales" (Gibson et al., 2009, p.229).

Por lo anterior, se encuentra una integración de la sociología de las organizaciones, con los enfoques administrativos mencionados en el apartado de la perspectiva económica - administrativa (humanístico, sistemático y de calidad), y con las tendencias actuales sobre las investigaciones de la calidad del servicio interno, dado que la sociología de las organizaciones considera que las empresas están conformadas por personas, las cuáles se ven afectadas en su comportamiento por la organización, revistiendo interés en el diseño del modelo teórico para el estudio de la calidad del servicio interno, el señalamiento entre la diferencia del comportamiento individual y el grupal, conformándose éstos últimos a partir de sus necesidades.

\subsubsection{La calidad del senvicio interno dentro de las empresas turísticas en el marco del desarrollo social sustentable}

Con el desarrollo sustentable se encuentra el punto de unión entre las disciplinas revisadas (económico administrativas, marketing y la sociología de las organizaciones) y el concepto de calidad del servicio interno, ya que en un marco de sustentabilidad, las empresas deben de generar actividades para el bienestar y el progreso de la población en armonía con la preservación del medio ambiente, siendo pues, la instrumentación de la calidad del servicio interno un motor para ello.

El concepto de internal service quality cobra vital importancia en el sector hotelero, ya que como lo menciona Pittaway, Carmouche y Chell (1998), la industria de la hospitalidad es una industria de la gente $y$ es frecuentemente caracterizada como heterogénea debido al gran número de consumidores directamente involucrados en la producción y proceso del servicio.

Uno de los temas que se pone de manifiesto en la calidad del servicio interno de la mano con el desarrollo sustentable en el campo de la industria hotelera, son las condiciones laborales, ya que éstas van en decadencia sin presentar aún una preocupación real por parte del sector empresarial. Pero además en el campo de la investigación existe una escases de este tema (Sánchez y Olivarria, 2016).

Como lo expresa la Organización Internacional del Trabajo (OIT) (2010), las condiciones de trabajo en la actividad turística son diferentes a otros sectores, siendo éstas, trabajos antisociales e irregulares, los cuáles aumentan la presión sobre los trabajadores con responsabilidades familiares, especialmente sobre las mujeres, que asumen la mayor parte de las tareas de cuidado de los niños y ancianos, así como las labores domésticas. Además, los trabajadores dependen fundamentalmente de los miembros de la familia o de los servicios privados o públicos para cumplir con las obligaciones relacionadas con el cuidado infantil.

Esta organización también vislumbra las siguientes problemáticas enmarcadas en condiciones laborales del sector hotelero: inseguridad laboral, salarios comparativamente bajos (con frecuencia por debajo de la media nacional), inestabilidad laboral, reducidas oportunidades de desarrollo profesional, elevado nivel de empleo en régimen de subcontratación y de externalización, así como el alto índice de rotación y de contratos a tiempo parcial, ocasionales y flexibles. En lo que respecta al aumento salarial, esta organización señala que es vulnerable a los cambios externos como la inflación.

En este sentido, en el contexto mexicano, específicamente en la zona de Toluca-Metepec, los autores Juárez, Lechuga, Méndez y López (2013) señalan que las condiciones de trabajo del sector hotelero se acercan a la precariedad, y se observa una creciente flexibilidad laboral. Igualmente mencionan, que si bien un grupo considerable de trabajadores poseen prestaciones por encima del promedio, existe una estrechez en el nivel salarial y poco margen de oportunidad para acrecentar los niveles de calidad de vida. 
De ahí el interés por incorporar las condiciones laborales como categoría de análisis en el ambiente de la calidad del servicio interno, ya que si bien es cierto que México ocupa el noveno lugar a nivel mundial en llegadas de turistas (Organización Mundial de Turismo, 2016), también es una realidad que esto no se ve reflejado en su población. Al respecto Palafox, Zizumbo y Arriaga (2010) establecen que "el hecho de que un país cuente con un sector de servicios de gran magnitud, no necesariamente indica su grado de avance económico, es decir, el sector servicios de un país pobre podría ser grande, pero la calidad de su empleo baja, como es el caso de México, en donde las condiciones laborales continúan en detrimento tanto en el ámbito turístico como a nivel general".

Pero las condiciones laborales traen consigo situaciones personales que dependerán de una adaptabilidad a partir del contexto en el que se viva, por ello las siguientes dos categorías a analizar dentro del modelo teórico de la calidad del servicio desde una perspectiva social sustentable son: las situaciones laborales y el contexto local, ya que el desarrollo sustentable va más allá de tres dimensiones (económica, social y ambiental), éste tiene que ver con el desarrollo de la población, con un desarrollo con calidad en el crecimiento a través del incremento de los niveles de bienestar y reducción de las desigualdades socioeconómicas.

Desde 1999 con el modelo teórico de Chu-Hua Kuei, se ponía de manifiesto que uno de los factores más importantes para determinar la calidad del servicio interno de una organización era el ambiente, por lo que las categorías de análisis antes mencionadas, son el punto central a indagar en ese ambiente. Las investigaciones sobre el avance del concepto demarcan interés sobre estudios en relación al comportamiento del recurso humano, y específicamente en la industria de la hospitalidad, en el estudio de Wildes (2007), el autor utiliza la teoría de la psicología social como la base de la investigación en relación al estereotipo del mesero, a la mercadotecnia como las estrategias que vinculan la imagen percibida a la satisfacción del cliente, y la calidad del servicio interno como la literatura que ve al empleado como un valor en vez de un costo, proponiendo el manejo de la satisfacción del empleado. Por lo que abordar este concepto en la investigación, específicamente en las empresas turística, contribuye en el avance del conocimiento.

\section{MODELO TEÓRICO}

El objetivo del presente modelo teórico, es observar a la calidad del servicio interno desde diferentes enfoques (económico - administrativo, marketing, sociología de las organizaciones) integrándolos en un solo modelo derivado de encontrar un punto de conjugación entre ambos: la satisfacción del trabajador.

Como punto de partida, está la sociología de las organizaciones, al establecer a la empresa como un ente social y abierto influida por factores internos y externos entre los cuáles se encuentran las tres categorías de análisis supuestas en el ambiente de las empresas turísticas: condiciones laborales, situaciones personales y contexto local.

Ahora bien, el ambiente de la organización es reflejado en el comportamiento organizacional tanto de una forma individual como colectiva, poniéndose de manifiesto a través de ello de acuerdo a la perspectiva económica - administrativa: la satisfacción del trabajador y por ende su productividad.

Desde el mismo enfoque se menciona que como colectivo, las personas principalmente se unen de acuerdo a sus necesidades, siendo estás desde las básicas hasta las de status, poder o afiliación. Por lo que la motivación y el liderazgo que se empleen tanto del mismo grupo como de la empresa, influyen en el comportamiento organizacional grupal.

Al mismo tiempo, trabajar bajo una administración de calidad total como lo es hoy en día en las empresas de servicios, especialmente en los hoteles internacionales, supone una organización en donde el pilar es una cultura de calidad, la cual coadyuva junto con las estrategias de marketing interno a la mejora del comportamiento organizacional grupal a partir de sus necesidades, y desde la filosofía de la calidad del servicio, a actitudes hacia el servicio, y una calidad en los servicios recibidos al interior de la empresa.

Por lo que la calidad del servicio interno significa para las empresas turísticas, una forma de trabajo en el marco del desarrollo sustentable, vinculada con la dimensión de calidad de vida, estableciendo de esta manera el siguiente modelo teórico (Figura 1). Con esta visión integradora se propone observar como objeto de estudio la calidad del servicio interno bajo las tres categorías de análisis en relación a las siguientes interrogantes: 
- ¿Cuáles son las situaciones personales y el contexto del trabajador como factores externos, que se insertan en el ambiente de la organización?

- ¿Cuáles son las condiciones laborales que afectan en lo colectivo el comportamiento organizacional colectivo?

- ¿Cuáles son las condiciones laborales que afectan las situaciones personales de los trabajadores?

- ¿Cuáles son las condiciones laborales, las situaciones personales y el contexto de los trabajadores que están presentes o se relacionan con la calidad del servicio interno?
Una parte importante dentro de este análisis integrador mutidisciplinar, es considerar que la industria hotelera internacional, supone la aplicación de uno o varios modelos de gestión de calidad, por lo que en ellos se trabaja sobre una filosofía de calidad y una instrumentación al respecto, y que además, se implementan estrategias de marketing interno conllevando a un ambiente de calidad con un resultado de satisfacción del trabajadory una productividad de la empresa.

Figura 1. Modelo Teórico de la Calidad del senvicio interno desde la perspectiva social sustentable.

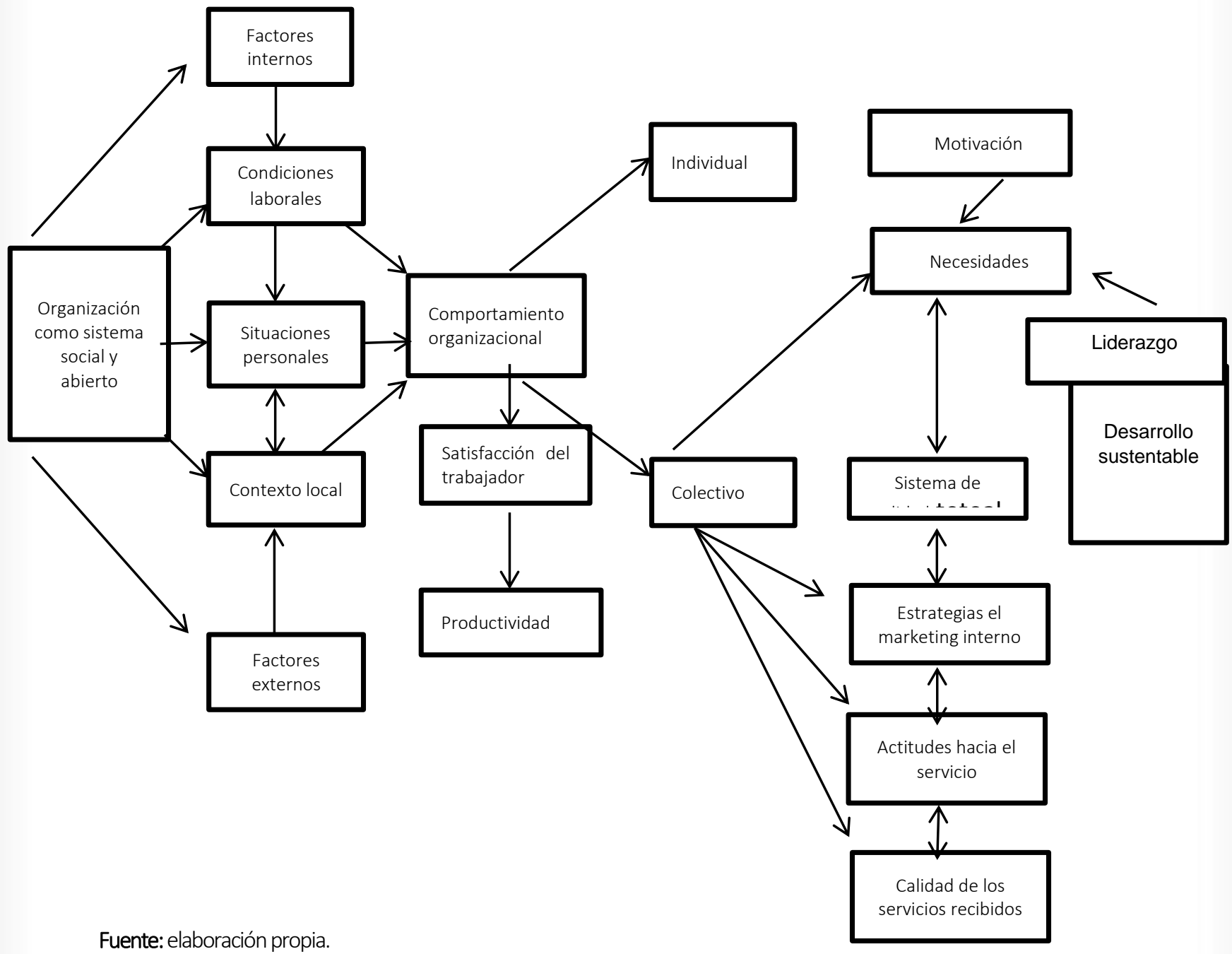

Con lo anterior se atenderían las recomendaciones de las investigaciones más recientes que van en la directriz de indagar a profundidad aspectos del comportamiento del trabajador, sobre todo en variables exógenas como factores culturales, y variables sociodemográficas como: edad, género, nivel educacional, etc.; así como distinguir dentro del estudio entre efectos a nivel individual y efectos a nivel grupal en los multi-niveles de una organización.

Como se mencionó desde un inicio, la comprensión de la calidad del servicio interno es 
compleja, derivado de la cantidad de variables que contiene en su análisis. Con este modelo se busca integrar las disciplinas que a la fecha han abordado el estudio de este constructo como son: la económica administrativa, el marketing y la sociología de las organizaciones a la par de las necesidades actuales en las investigaciones, y con ello avanzar en el campo del conocimiento.

\section{ALCANCE Y LIMITACICIONES}

El diseño del modelo teórico fue pensado principalmente en la necesidad de investigaciones cualitativas que detecten los factores que están afectando el comportamiento del recurso humano dentro de la organización. El objetivo del planteamiento del modelo teórico es contar con el sustento teórico para el análisis de la calidad del servicio interno desde una perspectiva social sustentable.

Por lo anterior, el modelo puede ser considerado para analizar la calidad del servicio interno en hoteles internacionales bajo características similares en relación al contexto de los trabajadores, o podría considerarse un cambio en las categorías, siempre y cuando éstas, sean relacionadas con el comportamiento de los trabajadores al interior de la empresa, y vinculadas a un entorno externo o interno.

Las categorías de análisis sobre las que se basa el modelo, fueron determinadas a partir de las condiciones actuales del sector turismo en México, tanto en cuestiones laborales como en personales, en uno de los principales destinos de ese país: Playa del Carmen.

Derivado de la cantidad de variables que se pueden relacionar con el concepto calidad del servicio interno, no se precisan variables comprobadas en la satisfacción del trabajador y el comportamiento organizacional, así como con las necesidades de las personas y la motivación laboral, sino que se toman conceptos globales con el objetivo de indagar a profundidad en relación con la calidad del servicio interno. Sin embargo, las posibles líneas de investigación pueden estar a partir de esas relaciones comprabas, y la calidad del servicio interno tanto de forma cualitativa como cuantitativa.

Por último, por el tipo de empresas al que está dirigido este modelo (hoteles internacionales), se hace énfasis que en el análisis del concepto se debe de considerar que dichas empresas ya emplean algún tipo de sistema de gestión de calidad.

\section{CONCLUSIONES}

Estudiar el concepto de calidad en el servicio interno, se enmarca en una serie de corrientes disciplinares, que con el paso del tiempo se han conjugado para comprender desde varias perspectivas por lo que se puede afirmar que actualmente este término es complejo e interdisciplinar.

La calidad del servicio interno se identifica dentro de una organización, por lo que la forma de administración de la empresa influye en la forma en la que se desarrolla la calidad del servicio al interior, por ello se parte de la administración para su estudio. Desde el enfoque humanístico en la administración se hace ver la importancia del factor humano dentro de la empresa, la búsqueda para comprender su comportamiento en lo individual y en lo colectivo se vuelve parte del quehacer de la administración, siendo la motivación y el liderazgo parte de sus elementos.

Al mismo tiempo, desde un enfoque sistémico de la administración se demuestra que el ambiente externo que envuelve a la empresa proporciona información para el desempeño de la misma (considerada como el sistema), por lo que la empresa debe de adaptarse al ambiente, al estar ambos interrelacionados y ser interdependientes. Además, dentro de este enfoque se estudia a la calidad desde un enfoque estadístico, centrando su interés en su medición y análisis. Con el paso del tiempo se ve a la calidad como una ventaja competitiva, y no solo como una orientación estadística enfocada a la mejora de los procesos productivos de la empresa. EI enfoque sistémico de la administración se entrelaza con el enfoque humanístico para analizar a la calidad del servicio interno desde un contexto local, permitiendo identificar las influencias del ambiente en donde se desempeñan las organizaciones y de qué forma son tratadas al interior de la empresa, para determinar cuáles impactan en la calidad del servicio al interior de la empresa. Por su parte, la administración por calidad se integra a estos dos enfoques al instrumentar la calidad utilizando conceptos y técnicas de la calidad total al interior de la empresa.

Desde la perspectiva del marketing se han generado varios modelos teóricos enfocados a la comprensión de la calidad del servicio enfocada al cliente, la preocupación por ofrecer una alta calidad al cliente llevó a los académicos a observar hacia el 
interior de la empresa a través del trabajador como conductor del servicio. La investigación científica sobre las dimensiones que componen a la calidad del servicio interno y cómo se enlazan con la calidad que se ofrece al cliente, han sido de ayuda para que las organizaciones puedan instrumentarlos y con ello evaluar sus operaciones. Tanto la calidad del servicio como el marketing interno forman una base para el estudio de la calidad del servicio interno, ya que abordan aspectos filosóficos similares como: la importancia del recurso humano en el proceso productivo de la empresa, la medición de la satisfacción del servicio al cliente, y la importancia de ofrecer trabajos que contribuyan a una satisfacción del empleado.

Por último, al estudio de la calidad del servicio interno se le une la sociología de las organizaciones y el desarrollo sustentable, la primera siendo el eje central en los enfoques administrativos antes mencionados y dirigida hacia la comprensión de la conducta de las personas a través del comportamiento organizacional; $y$ la segunda, por las necesidades actuales de las empresas a nivel mundial de mantener un equilibrio con el ambiente, la economía y la sociedad, revistiendo especial interés en las empresas turísticas.

Por lo anterior, la revisión literaria en la búsqueda de la comprensión de la calidad del servicio interno ha descifrado un hilo conductor desde la perspectiva económica - administrativa, a la del marketing y la sociología de las organizaciones en el marco de un desarrollo sustentable. Las necesidades actuales tanto en el saber cómo en la práctica, encaminan este enfoque a identificar las situaciones personales y las condiciones laborales de los trabajadores, impulsando con ello estudios sobre la satisfacción del trabajador en el contexto en el que se desenvuelven, en el marco de una calidad del servicio interno desde una perspectiva social colectiva sustentable en las empresas turísticas.

\section{REFERENCIAS}

Anderson, E.W., Fornell, C. y Lehmann, D.R. (1994). Customer satisfaction, market share and profitability: findings from Sweden. Journal of Marketing, 58 (3), 53-66.

Balomori, G. y Flores, J. (2014). MECSI: Modelo para evaluar la calidad del servicio interno. InnOvacionOnes de negocios, 11(22), 191-213.

Cantú, H. (2011). Desarrollo de una cultura de calidad. México: McGrawHill.
Caruna, A. y Pitt, L. (1997). An internal measure of service quality and the link between service quality and business performance. European Journal of marketing, 31(8), 604-616.

De Val Pardo, I. (1997). Organizar, acción y efecto. Madrid: ESIC.

Dumitrescu, L., Cetina, I. y Pentescu, A. (2012). Employee satisfaction measurement - part of internal marketing. Review of International Comparative Management, 13, 37-48.

Chen, Wen-Jung (2013). Factors influencing internal service quality at international tourist hotel. Internacional Journal of Hospitality managment, 35, 152-160.

Chiavenato, I. (2014). Introducción a la teoría general de la administración. México: McGraw Hill.

Chi, Ch. \& Gursoy, D. (2009). Employee satisfaction, customer satisfaction, and financial performance: An empirical examination. International Journal of Hospitality Management, 28, 245-253.

Chu-Hua, K. (1999). Internal service quality - an empirical assessment. International Journal of Quality \& Reliability Management, 16(8), 783-791.

Christopher, M., Payne, A. y Payne, A. (1994). Marketing relacional: integrando la calidad, el servicio al cliente yel marketing. Madrid: Díaz de Santos, S.A. Recuperado de:

https://books.google.com.mx/books?id=YMfCGu3ByMC\&printsec=frontcover\&dq=marketing+relacional \&hl=es\&sa=X\&ved=0ahUKEwi8 ani27PTAhUB6YMK Hc yAbYQ6AEIKjAB\#v=onepage\& $q=$ =marketing\%20rel acional\&f=false

Croes, R y Rivera, M. (2015). Tourism and Human Development. Revista Latino-Americana Turismología, julio, 1 (2), 17-29. Recuperado de: https://rlaturismologia.ufff.emnuvens.com.br/rlaturis mologia/article/view/25/18.

Fadil, H., Singh, K. y Joseph, C. (2016). Influence of organizational innovation towards internal service quality in MBKS. Procedia - Social and Behavioral Sciences, 224, 317-324.

Finn, D., Baker, J., Marshall, G. y Anderson, R. (1996). Total quality management and internal customers: Measuring internal service quality. Journal of Marketing Theory and Practice, Summer, 35-50.

Frost, F. y Kumar, M. (2001). Service quality between internal customer and internal suppliers in an international airline. International Journal of Quality \& Reliability Management, 18, 371-386.

Gibson, J., Ivancevich, J., Donnelly J. y Konopaske, R. (2013). Organizaciones comportamiento, estructura $y$ procesos. México: McGraw Hill.

Gounaris, S. (2006). Internal market orientation and its measurement. Journal of Business Research, 59, 432-448.

Gunawardene, G. (2009). Relationship between dimensions of internal service quality and the nature of the internal service encounter - A study in the healthcare industry. California Journal of Operations Management. 7(1), 2130. 
Handel, M. (2003). The sociology of organization: classic, contemporary, and critical readings. London: SAGE Publications.

Recuperado

de:

https://books.google.com.mx/books?id=zUZ3HEi4dXI c\&printsec=frontcover\&dq=sociology+of+organizatio ns\&hl=es\&sa=X\&ved=0ahUKEwim78zmwsLTAhVJxo MKHQPyC5YQ6AElljAA\#v=onepage\&q=sociology $\% 20$ of\%20organizations\&f=false

Heskett, J., Jones, T., Loveman, G., Sasser, W. y Schlesinger, L. (1994). Putting the service-profit chain to work. Harvard Business Review, March-April, 164-170.

Juárez, R., Lechuga, J.J., Méndez, M. y López, E. (2013). Condiciones laborales en las empresas hoteleras de la Zona Toluca-Metepec-Lerma: su impacto en el desarrollo económico local. Universidad Autónoma del Estado de México. Recuperado de: http://ri.uaemex.mx/handle/20.500.11799/49664

Jun, M. y Cai, S. (2010). Examining the relationships between internal servisse quality and its dimensions, and internal customer satisfaction. Total Quality Management, 21(2), 205-223.

Kralj, A. y David, S. (2010). Service climate and customer satisfaction in a casino hotel: an exploratory case study. International Journal of Hospitality Management, 29, 711-719.

Large, R.O. y König, T. (2009). A gap model of purchasing's internal service quality: Concept, case study and internal survey. Journal of Purchasing \& Supply Management, 15, 24-32.

Larios, A. y Gonzalez, A. (2017). La calidad del servicio interno en el sector hotelero: objeto e instrumento de estudio multidisciplinar. Teoría y Praxis, 22, 113-136.

Lings, I. (2004). Internal market orientation Construct and consequences. Journal of Business Research. 57, 405413.

Luna, A. (2015). Proceso administrativo. México: Patria.

Newstrom, J. (2011). Comportamiento humano en el trabajo. México: McGrawHill.

Organización Internacional del Trabajo (2010). Cambios y desafios en el sector de la hotelería y el turismo. Recuperado

http://www.ilo.org/wcmsp5/groups/public/ed dialogue/-

sector/documents/meetingdocument/wcms 162207 .pdf Recuperado 27/07/2016

Organización Mundial del Turismo (2016). Barómetro OMT del Turismo Mundial. OMT. 4 (2016). Recuperado de: http://observatorituristic.aralleida.com/ftp/document s/Estadistiques/UNWTO\%20-

\%200rganitzaci\%C3\%B3\%20Mundial\%20del\%20Turis me/OMT TEND Maig2016 Annex cast.pdf

Palafox, A., Zizumbo, L. y Arriaga, E. (2010). El turismo como eje de acumulación: caso del sector hotelero en México. Multiciencias. 10(2), 193-201.

Pantouvakis, A. (2011). Internal service quality and job satisfaction synergies for performance improvement: Some evidence from a B2B enviroment. Journal of
Targeting, Measurement and Analysis for Marketing, 19, 11-22.

Pantouvakis, A. y Mpogiatzidis, P. (2013). The impact of internal service quality and learning organization on clinical leaders' job satisfaction in hospital care service. Leader in Health Service, 26(1), 34-49. Doi: 10.1108/17511871311291714

Parasuraman, A., Zeithmal, V. y Berry, L. (1985). A conceptual model of service quality and its implications for future research. Journal of Marketing, 49, 41-50.

Parasuraman, A., Zeithmal, V. y Berry L. (1988). SERVQUAL: a multiple-ltem scale for measuring consumer perceptions of service of service quality. Journal of Retailing, 64(1), 12-40.

Peinado, llenia y García, Alejandro (2016). Tendencias y posicionamiento de la actividad turística: algunos problemas y retos para México. Revista LatinoAmericana Turismología, julio, 2 (2), 77-89. Recuperado de: https://rlaturismologia.ufff.emnuvens.com.br/rlaturis mologia/article/view/67/44.

Pittaway, L., Carmouche, R. y Chell, E. (1998). The way forward: leadership research in the hospitality industry. Hospitality Management, 17, 407-426.

Robbins, S. (1998). Fundamentos de comportamiento organizacional. México: Prentice Hall.

Romero, I. y Ledo, M. (2008). Elevación de la calidad en los servicios a partir de la interrelación clientes internos y externos. Industrial, XXIX (3), 1-7.

Rue, L. y Byars, LL. (2013). Administración: Teoría y aplicaciones. México: Alfaomega.

Sánchez, V. y Olivarría, C. (2016). Desafíos y derroteros de los estudios laborales en turismo México: el tema de la flexibilidad laboral. Revista Latino-Americana Turismología,julio, 2 (2), 49-58. Recuperado de: https://rlaturismologia.ufjf.emnuvens.com.br/rlaturis mologia/article/view/49/43

Sasser, E. y Arbeit, S. (1976). Selling jobs in the service sector. Business Horizons, June, 61-65.

Sharma, P., Chuen, T., Kingshott, R. (2016). Internal service quality as a driver of employee satisfaction, commitment and performance. Journal of service management, 27(5), 773-797. Doi: 10.1108/JOSM-102015-0294

To, W., Martin, Jr., E. y Yu, T. (2015). Effect of management commitment to internal marketing on employee work atitude. International Journal of Hospitality Management, 45, 14-21.

Turkoz, I. y Akyol, A. (2008). Internal marketing and hotel performance. Anatolla: An International Journal of Tourism and Hospitality Research, 19, 149-177.

Umamaheswari, J. (2014). Exploring internal service quality in a manufacturing organization-A study in Lucus TVS, Chennai. Procedia Economics and Finance. 11, 710-725.

Vanniarajan, T. y Subbash, B. (2011). Internal service quality and its consequences in commerical banks: A HR perspective. Global Management Review, 6, 42-57. 
Velázquez, G. (2008). Sociología de las organizaciones. Limusa:México. Recuperado de: https://books.google.com.mx/books?id=xOr9HK74n1 wC\&printsec=frontcover\&dq=sociolog\%C3\%ADa+de +las+organizaciones\&hl=es\&sa=X\&ved=0ahUKEwitqt KssLTAhVJ4IMKHX9YC7sQ6AEIOjAF\#v=onepage\&q= sociolog\%C3\%ADa\%20de\%20las\%20organizaciones\& $\mathrm{f}=$ false

Wildes, V. (2007). Attracting and retaining food servers: How internal service quality moderates occupational stigma. Hospitality management, 26, 4-19.

Yee, R., Yeung, A. \& Chen, E. (2008). The impact of employee satisfaction on quality and profitability in high-contact service industries. Journal of operations management, 26, 651-668.

Yee, R., Yeung, A. y Cheng, T. (2011). The service-profit chain: An empirical analysis in high contact servicee industries. Int J. Production Economics, 130, 236-245.

Processo Editorial / Editorial Process

Editor Chefe/Editor-in-chief: PhD Thiago D. Pimentel (UFJF).

Recebido em 217de Julho de 2017; aceito em 15 de Dezembro de 2017; publicado online 05 de Fevereiro de 2018. Received on July 27, 2017; accepted on December 15, 2017, published online on February 05, 2018. Texto original/ Original paper. Sistema de revisão cega por pares / Double blind review system. 